\title{
A novel power-efficient scheme asymmetrically and symmetrically clipping optical (ASCO)-OFDM for IM/DD optical systems
}

\author{
Nan $\mathrm{Wu}^{*}$ and Yeheskel Bar-Ness
}

\begin{abstract}
In this paper, we propose a novel scheme of orthogonal frequency division multiplexing (OFDM) for intensity modulation direct detection (IM/DD) optical systems. By using this novel scheme of an OFDM optical system, not only odd subcarriers but also even subcarriers can be modulated to transmit a clipping optical signal. A conventional asymmetrically clipping optical (ACO)-OFDM is applied to modulate odd subcarriers while even subcarriers are modulated by a novel technique called a symmetrically clipping optical (SCO)-OFDM. Although both the asymmetrically clipping noise caused by ACO-OFDM and the symmetrically clipping noise caused by SCO-OFDM fall onto the even subcarriers, the former interference can be estimated and removed at the receiver. Thus, SCO-OFDM symbols carried on the even subcarriers can be recovered by subtracting the estimated ACO-OFDM clipping noise from the received signal. Then the SCO-OFDM clipping noise can be removed by subtraction due to its special transmission format. Note that no DC bias added on all subcarriers makes this novel scheme achieve better performance in terms of both power efficiency and symbol error rate (SER).
\end{abstract}

Keywords: OFDM; Power efficient; IM/DD; Wireless optical systems

\section{Introduction}

Optical wireless systems with intensity modulation direct detection (IM/DD) have been extensively studied in recent decades [1-3]. They can be effective alternatives to radio frequency (RF) wireless systems for indoor high-speed data transmission. Compared to RF communication, optical wireless communication (OWC) offers unlimited transmission bandwidth and efficient spatial diversity achieved with short carrier wavelengths and a large area detector [4]. Multipath distortion, especially in an indoor environment, caused by reflection from walls or furniture, severely affects the transmission quality of an optical signal. Orthogonal frequency division multiplexing (OFDM) [5] is a promising modulation technique and has been widely used to combat inter-symbolinterference (ISI) resulting from multipath propagation in

*Correspondence: nw27@njit.edu

Department of Electrical and Computer Engineering, New Jersey Institute of Technology, Newark, NJ 07102, USA
RF communication. The technique of OFDM has been successfully applied in IM/DD optical wireless systems, such as a DC-biased optical OFDM (DCO-OFDM) [6] and an asymmetrically clipped optical OFDM (ACOOFDM) $[7,8]$. Combining the techniques of the previous two schemes, an asymmetrically clipped DC-biased optical OFDM (ADO-OFDM) [9] has been developed, and it is more efficient in terms of bandwidth and optical power. Also, there are some other full spectrally efficient optical OFDM systems, such as hybrid ACO-OFDM [10] and polar-OFDM [11].

In IM/DD optical wireless systems, the information data stream is modulated into the intensity of optical carriers. Thus, only real and non-negative values can be used to realize the intensity modulation. In order to obtain a real signal, we apply an inverse fast Fourier transform (IFFT) to convert blocks of Hermitian symmetry complex symbols. For the non-negative requirement of the transmitted optical signals, two mainstream approaches are extensively adopted, adding a DC bias and clipping. 
In DCO-OFDM, a DC bias is added to eliminate the negative values in the transmitted signal. However, the performance of DCO-OFDM strongly depends on the DC bias level [12]. If the added DC bias cannot exceed the negative peak, the remaining negative values must be clipped to zero, then all subcarriers will be affected by the clipping noise. If the DC bias is larger than the negative peak, it makes the DCO-OFDM inefficient in terms of optical power. The advantage of DCO-OFDM is bandwidth efficiency because both odd and even subcarriers are used to carry data. In ACO-OFDM, all the negative values are clipped to zero, leaving the positives. In [13], we found that the clipping approach reduces the amplitude of the original symbols by half, and the clipping noise falls onto the even subcarriers without distorting the data on the odd subcarriers. Thus, only odd subcarriers are used to carry data in ACO-OFDM. Compared to DCOOFDM, ACO-OFDM is more optical power efficient, but its bandwidth is as twice that of DCO-OFDM.

In order to maintain the advantages and avoid the drawbacks of the previous two optical systems, a combination scheme called ADO-OFDM has been designed. ACOOFDM symbols modulate odd subcarriers while DCOOFDM symbols modulate even subcarriers. The negative values generated by ACO-OFDM and DCO-OFDM are separately clipped to zero. Then, the sum of the remaining two non-negative signals is transmitted by a light emitting diode (LED) $[14,15]$. ACO-OFDM symbols can be easily recovered by detecting the data carried on the odd subcarriers. The ACO-OFDM clipping noise can be estimated from the received ACO-OFDM signals. By adding a moderate $\mathrm{DC}$ bias at the transmitter, the clipping noise caused by DCO-OFDM is negligible compared to the wanted signal. Finally, DCO-OFDM symbols are successfully recovered by subtracting the estimated ACOOFDM clipping noise from the received DCO-OFDM signals.

Adding a DC bias is a simple and direct approach, but it leads to a large waste of optical power. A full spectrally efficient optical system, hybrid ACO-OFDM, is proposed by Bilal Ranjha and Mohsen Kavehrad. Without using the DC bias, it is more power efficient than ADO-OFDM. The ideas for the system design of the hybrid ACO-OFDM and our proposed system are similar. For both, the odd subcarriers are used to carry ACO-OFDM symbols, which are drawn from quadrature amplitude modulation (QAM) constellations. The differences are that the hybrid ACOOFDM uses the imaginary parts of the even subcarriers to carry PAM-DMT symbols, while we still map the QAM symbols onto the even subcarriers. We believe that our proposed system will be better than the hybrid ACOOFDM because the average power of QAM symbols is much smaller than that of PAM symbols in the same level of constellation case.
So far, the Hermitian symmetry constraint is usually enforced on the signal vector for obtaining real signals. However, Hany Elgala and Thomas D. C. Little provided another scheme, polar-OFDM (P-OFDM), to generate real unipolar signals without this constraint. The QAM symbols are only mapped onto the even subcarriers. After taken by IFFT, the complex signal vector satisfies the half-wave even symmetry. Then, the authors applied the cartesian-to-polar operation to convert the complex form into the exponential form. The amplitude and the phase values of different samples are transmitted. At the receiver, Hany Elgala and Thomas D. C. Little used the reverse operation to recover the original complex symbols. The designing ideas of P-OFDM and our proposed system are quite different. The main difference depends on the use of the Hermitian symmetry constraint.

In this paper, we propose a novel scheme, called an asymmetrically and symmetrically clipping optical (ASCO)-OFDM, to simultaneously transmit ACO-OFDM symbols on the odd subcarriers and symmetrically clipping optical (SCO)-OFDM symbols on the even subcarriers. If the length of an ACO-OFDM signal is $2 \mathrm{~N}$, an ACO-OFDM symbol that consists of only odd subcarriers has the property of $x(n)=-x(n+N)[13,16]$. However, an OFDM symbol converted from only even subcarriers satisfies the property that $x(n)=x(n+N)$, which is proved in Appendix 1. After clipping, the same negative values are removed in the first and in the second half. It is the reason that the non-negative signals are called SCO-OFDM signals. Fortunately, the SCO-OFDM clipping noise does not affect ACO-OFDM symbols because it falls onto the even subcarriers. We prove this claim in Appendix 2. By using the same operation in ADO-OFDM, we can recover ACO-OFDM symbols by extracting the data on the odd subcarriers. Also, the ACO-OFDM clipping noise can be accurately estimated from the recovered ACO-OFDM signals. The data on the even subcarriers are distorted by the ACO-OFDM clipping noise, the SCO-OFDM clipping noise, and all other noises. The details of reconstructing SCO-OFDM are discussed in Section 2.2. Since no DC bias is added to all subcarriers, this novel scheme exhibits better performances in terms of optical power and symbol error rate (SER) than ADO-OFDM does. Moreover, the existing technique [17] can be applied to improve the SER performance [18].

The rest of the paper is organized as follows: In Section 2.1, the structure of ADO-OFDM is briefly reviewed. In Section 2.2, we present the ASCO-OFDM system in detail. The probability density function (PDF) of the ASCO-OFDM signals is derived; the analysis of the average bit rate/normalized bandwidth and power/bit for both the ADO-OFDM and the ASCO-OFDM is presented in Section 3. Simulation results and performance comparisons between ADO-OFDM and ASCO-OFDM are given 
in Section 4. Finally, the conclusion of the paper is given in Section 5.

\section{System model}

\subsection{Review of asymmetrically clipped DC-biased optical (ADO)-OFDM}

Figure 1 shows the block diagram of ADO-OFDM. The input of the ADO-OFDM system is a block of $(N-1) \times 1$ complex data symbols $S=\left[S_{0}, S_{1}, \cdots, S_{N-2}\right]^{T}$ mapped from constellations such as 4-QAM, 16-QAM, or 64QAM. In order to obtain a real signal, this block of complex data symbols is combined with its conjugate sequence $S^{*}=\left[S_{0}^{*}, S_{1}^{*}, \cdots, S_{N-2}^{*}\right]^{T}$ in reverse order to form a $2(N-$ 1) $\times 1$ signal vector that has Hermitian symmetry as follows, $\left[S_{0}, S_{1}, \cdots, S_{N-2}, S_{N-2}^{*}, \cdots, S_{1}^{*}, S_{0}^{*}\right]^{T}$. Two zeroes are, respectively, inserted at the first and at the middle of this signal vector, which is shown as:

$$
X=\left[0, S_{0}, S_{1}, \cdots, S_{N-2}, 0, S_{N-2}^{*}, \cdots, S_{1}^{*}, S_{0}^{*}\right]^{T}
$$

Then $X$ is divided into two $2 N \times 1$ signal vectors, $X_{\text {odd }}$ and $X_{\text {even, }}$ that consist of the odd components and the even components of $X$, which are shown as:

$X_{\text {odd }}=\left[0, S_{0}, 0, S_{2}, \cdots, 0, S_{N-2}, 0, S_{N-2}^{*}, 0, \cdots, S_{2}^{*}, 0, S_{0}^{*}\right]^{T}$

$$
X_{\text {even }}=\left[0,0, S_{1}, 0 \cdots, S_{N-3}, 0,0,0, S_{N-3}^{*}, \cdots, 0, S_{1}^{*}, 0\right]^{T}
$$

Both $X_{\text {odd }}$ and $X_{\text {even }}$ are converted by a $2 N$-point IFFT to yield two real bipolar signal vectors, $x_{\text {odd }}$ and $x_{\text {even }}$, respectively. In an ACO-OFDM clipping block, the negative values in $x_{\text {odd }}$ are clipped to zero to produce a signal, $x_{\mathrm{ACO}}$, consisting of non-negative values. Another signal,
$x_{\mathrm{DCO}}$, is obtained by adding a DC bias to $x_{\text {even }}$ and setting the remaining negative values to zero. In order to avoid the DCO-OFDM clipping noise, a large DC bias should be added which makes the transmitted optical signal inefficient in terms of optical power. Thus, the performance of DCO-OFDM depends on the bias level. Generally, $x_{\text {even }}$ is modeled as a block of Gaussian random variables with mean zero and variance $E\left\{x_{\text {even }}^{2}\right\}$. The DC bias is denoted by $B_{\mathrm{DC}}$, which can be defined as follows: $B_{\mathrm{DC}}=\mu \sqrt{E\left\{x_{\text {even }}^{2}\right\}} \cdot 10 \log _{10}\left(\mu^{2}+1\right) d B$ is the decibel form of $B_{\mathrm{DC}}$, where $\mu$ is a proportionality constant. The transmitted signal $\tilde{x}_{\mathrm{ADO}}$ is the sum of $x_{\mathrm{ACO}}$ and $x_{\mathrm{DCO}}$ with the cyclic prefix (CP) attached.

An indoor optical wireless channel can be modeled as a multipath propagation channel [19]. The impulse response of this optical channel is given by $h(n)=$ $[h(0), h(1), \cdots, h(l)]$, where $l$ is the number of channel coefficients which determines the length of $\mathrm{CP}$. At the receiver, a photodiode is employed to detect the intensity of the received optical signal:

$$
\tilde{y}_{\mathrm{ADO}}(n)=\tilde{x}_{\mathrm{ADO}}(n) \otimes h(n)+w(n)
$$

which is the convolution of the incident optical signal and the optical channel plus noise. $w(n)$ represents the combination of shot noise and thermal noise, which can be approximated as additive white Gaussian noise. We assume that channel state information (CSI) is wellknown at the receiver. The output of a $2 N$-point fast Fourier transform (FFT), $Y_{\mathrm{ADO}}(k)$, is operated by a singletap equalizer in the frequency domain. $X_{\text {odd }}$ can be recovered by extracting the odd components of $Y$ followed by a hard or soft detection. By transforming $Y_{\text {odd }}$ into the time domain, an ACO-OFDM clipping noise estimation block reconstructs a reference signal $y_{\mathrm{ACO}}$. Finally, $y_{\mathrm{DCO}}$ is obtained by subtracting $y_{\mathrm{ACO}}$ from the reference signal

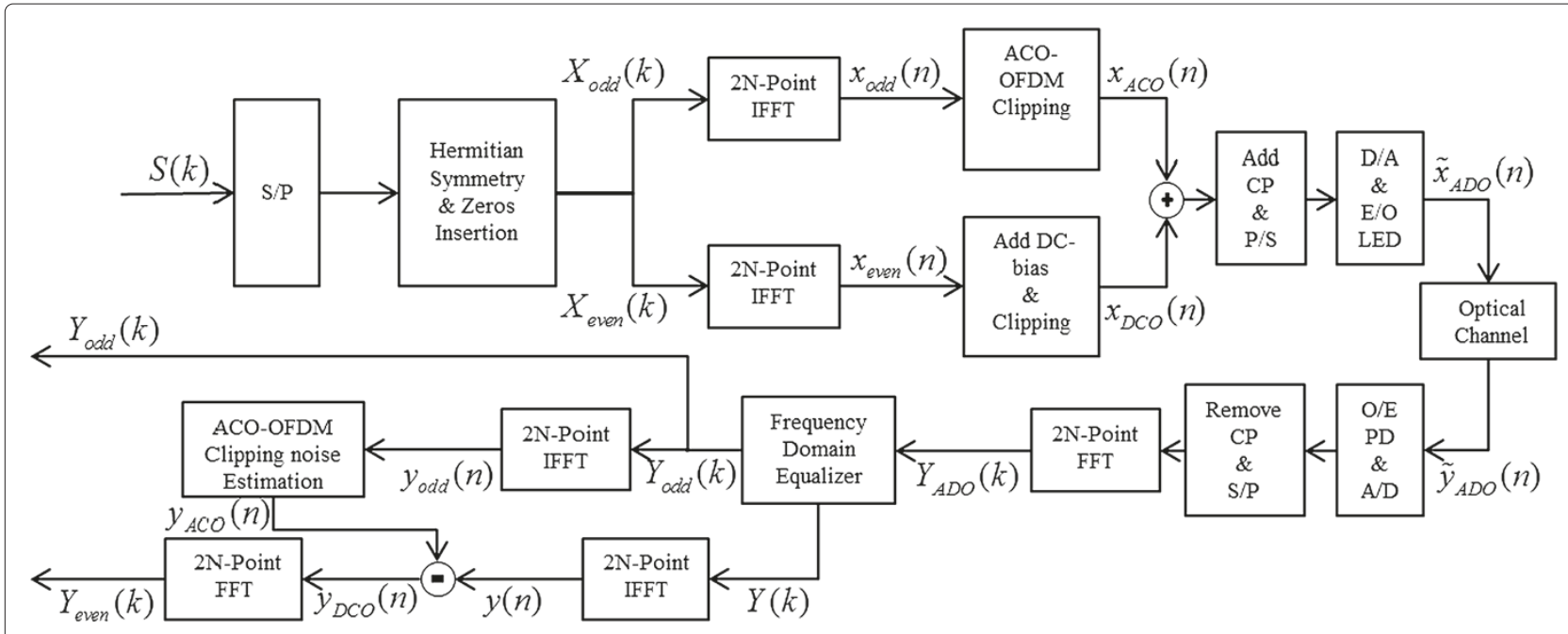

Figure 1 ADO-OFDM transmitter and receiver configuration. 
$y$, and it is transformed into the frequency domain to yield $Y_{\text {even }}$.

\subsection{Novel scheme - asymmetrically and symmetrically clipping optical (ASCO)-OFDM}

The block diagram of ASCO-OFDM is shown in Figure 2. Since the ASCO-OFDM system uses a novel transmission scheme, the input block of complex symbols is first separated into three parts, which have two $(N / 2) \times 1$ signal vectors and one $(N / 2-1) \times 1$ signal vector. Thus, the length of the input block is $3 N / 2-1$. Similarly, in order to obtain a real signal, we have to make the input of IFFT have the Hermitian symmetry. Two $(N / 2) \times 1$ signal vectors are, respectively, combined with their conjugate sequences and are inserted by zeroes into the even subcarriers to form two $2 N \times 1$ signal vectors, $X_{\text {odd }}^{i}$ and $X_{\text {odd }}^{j}$, which are given by:

$X_{\mathrm{odd}}^{i}=\left[0, S_{0}^{i}, 0, S_{1}^{i}, \cdots, 0, S_{N / 2}^{i}, 0, S_{N / 2}^{i *}, 0, \cdots, S_{1}^{i *}, 0, S_{0}^{i *}\right]^{T}$

$X_{\text {odd }}^{j}=\left[0, S_{0}^{j}, 0, S_{1}^{j}, \cdots, 0, S_{N / 2}^{j}, 0, S_{N / 2}^{j *}, 0, \cdots, S_{1}^{j *}, 0, S_{0}^{j *}\right]^{T}$

The $(N / 2-1) \times 1$ signal vector is defined in a similar way with zeroes inserted into the odd subcarriers to form a signal vector as follows:

$$
\begin{gathered}
X_{\mathrm{even}}=\left[0,0, S_{0}, 0, S_{1}, \cdots, S_{N / 2-1}, 0,0,0, S_{N / 2-1}^{*}, \cdots,\right. \\
\left.S_{1}^{*}, 0, S_{0}^{*}, 0\right]^{T}
\end{gathered}
$$

All three signal vectors $X_{\text {odd }}^{i}, X_{\text {odd }}^{j}$, and $X_{\text {even }}$ are constrained to the Hermitian symmetry. They are taken by a $2 N$-point IFFT to produce real bipolar signal vectors $x_{\text {odd }}^{i}$, $x_{\text {odd }}^{j}$, and $x_{\text {even }}$, respectively. To ensure the non-negative requirement of the transmitted signals, all negative values in $x_{\text {odd }}^{i}$ and $x_{\text {odd }}^{j}$ are clipped to zero to generate $x_{\text {odd }}^{i, c}$ and $x_{\text {odd }}^{j, c}$ which are given by:

$$
\begin{aligned}
& x_{\text {odd }}^{i, c}=0.5\left(x_{\text {odd }}^{i}+\left|x_{\text {odd }}^{i}\right|\right) \\
& x_{\text {odd }}^{j, c}=0.5\left(x_{\text {odd }}^{j}+\left|x_{\text {odd }}^{j}\right|\right)
\end{aligned}
$$

As each sample in $x_{\text {even }}$ is converted from even subcarriers, it has the relation that $x_{\text {even }}(n)=x_{\text {even }}(n+N)$. By clipping the negative values, half of the information carried in $x_{\text {even }}$ is lost. Thus, two signal vectors, $x_{\text {even }}^{c n}$ and $x_{\text {even }}^{c p}$, are generated for transmitting the information in $x_{\text {even }} \cdot x_{\text {even }}^{c n}$ represents that all negative values of $x_{\text {even }}$ are clipped to zero. $x_{\text {even }}^{c p}$ represents that all positive values of $x_{\text {even }}$ are clipped to zero and the remaining negative values are turned to positive. They are respectively given by:

$$
x_{\text {even }}^{c n}=0.5\left(x_{\text {even }}+\left|x_{\text {even }}\right|\right)
$$

$$
x_{\mathrm{even}}^{c p}=0.5\left(-x_{\mathrm{even}}+\left|x_{\mathrm{even}}\right|\right)
$$

Then, we construct a transmitted signal that consists of two consecutive sub-blocks, $x_{\mathrm{ASCO}}^{i}$ and $x_{\mathrm{ASCO}}^{j}$, which are given by:

$$
\begin{aligned}
x_{\mathrm{ASCO}}^{i} & =x_{\text {odd }}^{i, c}+x_{\text {even }}^{c n} \\
& =0.5\left(x_{\text {odd }}^{i}+\left|x_{\text {odd }}^{i}\right|+x_{\text {even }}+\left|x_{\text {even }}\right|\right)
\end{aligned}
$$

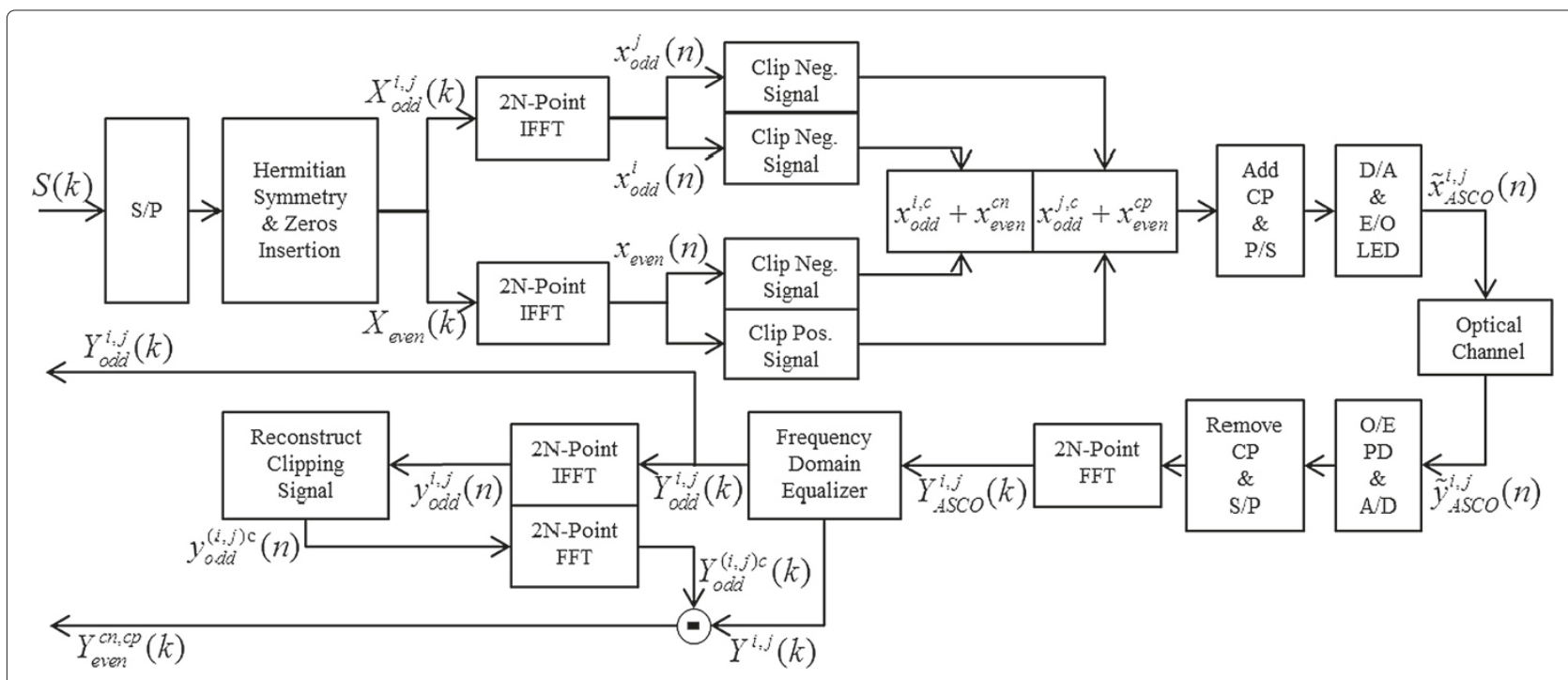

Figure 2 ASCO-OFDM transmitter and receiver configuration. 


$$
\begin{aligned}
x_{\mathrm{ASCO}}^{j} & =x_{\text {odd }}^{j, c}+x_{\text {even }}^{c p} \\
& =0.5\left(x_{\text {odd }}^{j}+\left|x_{\text {odd }}^{j}\right|-x_{\text {even }}+\left|x_{\text {even }}\right|\right)
\end{aligned}
$$

The transmitted signals, $x_{\mathrm{ASCO}}^{i}$ and $x_{\mathrm{ASCO}}^{j}$, with the cyclic prefix are denoted by $\tilde{x}_{\text {ASCO }}^{i}$ and $\tilde{x}_{\text {ASCO }}^{j}$. They are transmitted through an optical channel by an LED. The received signals are given by:

$$
\begin{aligned}
& \tilde{y}_{\mathrm{ASCO}}^{i}(n)=\tilde{x}_{\mathrm{ASCO}}^{i}(n) \otimes h(n)+w^{i}(n) \\
& \tilde{y}_{\mathrm{ASCO}}^{j}(n)=\tilde{x}_{\mathrm{ASCO}}^{j}(n) \otimes h(n)+w^{j}(n)
\end{aligned}
$$

where the impulse response of optical channel $h(n)$ is modeled as $h(n)=[h(0), h(1), \cdots \cdots, h(l)]$, and the sum of all noise, $w^{i}(n)$ or $w^{j}(n)$, is approximately modeled as additive white Gaussian noise. After removing the cyclic prefix, the arrival signals, $y_{\mathrm{ASCO}}^{i}$ and $y_{\mathrm{ASCO}}^{j}$, are, respectively, transformed by a $2 N$-point $\mathrm{FFT}$ into the frequency domain to yield $Y_{\mathrm{ASCO}}^{i}$ and $Y_{\mathrm{ASCO}}^{j}$. Then, a frequency domain equalizer with the knowledge of channel state information is applied to $Y_{\mathrm{ASCO}}^{i}$ and $Y_{\mathrm{ASCO}}^{j}$ to yield:

$$
\begin{aligned}
Y^{i} & =\left(\Lambda^{H} \Lambda+(\alpha / \mathrm{SNR}) I_{2 N}\right)^{-1} \Lambda^{H} Y_{\mathrm{ASCO}}^{i} \\
Y^{j} & =\left(\Lambda^{H} \Lambda+(\alpha / \mathrm{SNR}) I_{2 N}\right)^{-1} \Lambda^{H} Y_{\mathrm{ASCO}}^{j}
\end{aligned}
$$

where $\Lambda$ is a $2 N \times 2 N$ diagonal matrix whose diagonal is the $2 N$-point FFT of $h(n)$, and $\Lambda^{H}$ indicates the Hermitian matrix of $\Lambda$. The frequency domain equalizer can be a MMSE equalizer $(\alpha=1)$ or a ZF equalizer $(\alpha=0)$. $Y^{i}$ and $Y^{j}$ can also be shown in the frequency domain as follows:

$$
\begin{aligned}
Y^{i} & =X_{\text {odd }}^{i, c}+X_{\text {even }}^{c n} \\
& =0.5\left(X_{\text {odd }}^{i}+\left|X_{\text {odd }}^{i}\right|+X_{\text {even }}+\left|X_{\text {even }}\right|\right) \\
Y^{j} & =X_{\text {odd }}^{j, c}+X_{\text {even }}^{c p} \\
& =0.5\left(X_{\text {odd }}^{j}+\left|X_{\text {odd }}^{j}\right|-X_{\text {even }}+\left|X_{\text {even }}\right|\right)
\end{aligned}
$$

where $X_{\text {odd }}^{i}$ and $\left|X_{\text {odd }}^{i}\right|$ are the 2N-point FFT of $x_{\text {odd }}^{i}$ and $\left|x_{\text {odd }}^{i}\right|$. The other corresponding terms in Equations $12,13,18,19$ are similarly defined. Note that the symbols of $0.5 X_{\text {odd }}^{i}$ and $0.5 X_{\text {odd }}^{j}$ fall onto the odd subcarriers of $Y^{i}$ and $Y^{j}$, and the other symbols that consist of the remaining terms, $0.5\left(\left|X_{\text {odd }}^{i}\right|+X_{\text {even }}+\left|X_{\text {even }}\right|\right)$ and $0.5\left(\left|X_{\text {odd }}^{j}\right|-X_{\text {even }}+\left|X_{\text {even }}\right|\right)$, fall onto the even subcarriers of $Y^{i}$ and $Y^{j}$, respectively. Thus, the complex symbols of $X_{\text {odd }}^{i}$ and $X_{\text {odd }}^{j}$ can be easily recovered by extracting the symbols of $Y_{\text {odd }}^{i}$ and $Y_{\text {odd }}^{j}$, which are the odd components of $Y^{i}$ and $Y^{j}$, because the clipping noises resulting from $x_{\text {odd }}^{i, c}$ and $x_{\text {odd }}^{j, c}$ only affect the even components of $Y^{i}$ and $Y^{j}$. In order to accurately estimate the clipping noise, $Y_{\text {odd }}^{i}$ and $Y_{\text {odd }}^{j}$ are transformed into the time domain to yield two blocks of real bipolar signals $y_{\text {odd }}^{i}$ and $y_{\text {odd }}^{j}$, respectively. $y_{\text {odd }}^{i, c}$ and $y_{\text {odd }}^{j, c}$ are two blocks of clipping reference signals which are generated in the same way as $x_{\text {odd }}^{i, c}$ and $x_{o d d}^{j, c}$, respectively; then, they are transformed back into the frequency domain to yield $Y_{\text {odd }}^{i, c}$ and $Y_{\text {odd }}^{j, c}$, respectively. Compared to $Y_{\text {odd }}^{i}$ and $Y_{\text {odd }}^{j}, Y_{\text {odd }}^{i, c}$ and $Y_{\text {odd }}^{j, c}$ have the same symbol on the odd subcarriers, but the clipping noise appears on the even subcarriers. Therefore, $Y_{\text {even }}^{c n}$ and $Y_{\text {even }}^{c p}$ are obtained by subtracting $Y_{\text {odd }}^{i, c}$ and $Y_{\text {odd }}^{j, c}$ from $Y^{i}$ and $Y^{j}$, respectively, which are given by:

$$
\begin{aligned}
Y_{\text {even }}^{c n} & =Y^{i}-Y_{\text {odd }}^{i, c} \\
& =0.5\left(X_{\text {even }}+\left|X_{\text {even }}\right|\right) \\
Y_{\text {even }}^{c p} & =Y^{j}-Y_{\text {odd }}^{j, c} \\
& =0.5\left(-X_{\text {even }}+\left|X_{\text {even }}\right|\right)
\end{aligned}
$$

We note that $x_{\text {even }}=x_{\text {even }}^{c n}-x_{\text {even }}^{c p}$; hence, $Y_{\text {even }}$ can be estimated by the form:

$$
Y_{\mathrm{even}}=Y_{\mathrm{even}}^{c n}-Y_{\mathrm{even}}^{c p}
$$

\section{Signal analysis of ADO-OFDM and ASCO-OFDM}

In this section, we first review the PDF of ADO-OFDM signals. Then, the theoretical PDF of ASCO-OFDM signals is derived, and two simulated PDF results are compared with the theoretical PDF in Figure 3. Moreover, we analyze the average bit rate/normalized bandwidth of ASCO-OFDM and ADO-OFDM. Through calculating the total optical power and the information bit of the transmitted signal, we obtain the optical power per bit for both schemes.

\subsection{The PDF of ADO-OFDM}

The PDF of ADO-OFDM signals is the convolution of the PDF of ACO-OFDM signals and the PDF of DCO-OFDM signals, which is given by Eq. (28) in [9] because they have the relation that $x_{\mathrm{ADO}}(n)=x_{\mathrm{ACO}}(n)+x_{\mathrm{DCO}}(n)$. The PDFs of ACO-OFDM signals and DCO-OFDM signals are respectively given by [19-21]:

$$
\begin{aligned}
f_{x_{\mathrm{ACO}}(t)}(\chi) & =\frac{1}{\sqrt{2 \pi} \sigma_{A}} e^{-\frac{\chi^{2}}{2 \sigma_{A}^{2}}} u(\chi)+\frac{1}{2} \delta(\chi) \\
f_{x_{\mathrm{DCO}}(t)}(\chi) & =\frac{1}{\sqrt{2 \pi} \sigma_{D}} e^{-\frac{\left(\chi-B_{\mathrm{DC}}\right)^{2}}{2 \sigma_{D}^{2}}} u(\chi)+Q\left(\frac{B_{\mathrm{DC}}}{\sigma_{D}}\right) \delta(\chi)
\end{aligned}
$$

where $u(\chi)$ is the unit step function and $\delta(\chi)$ is the Dirac delta function. $\sigma_{A}$ is the standard deviation of unclipped signals in ACO-OFDM, which is given by $\sigma_{A}=E\left\{x_{\text {odd }}^{2}\right\}$. $\sigma_{D}$ is the standard deviation of DCO-OFDM signals, 


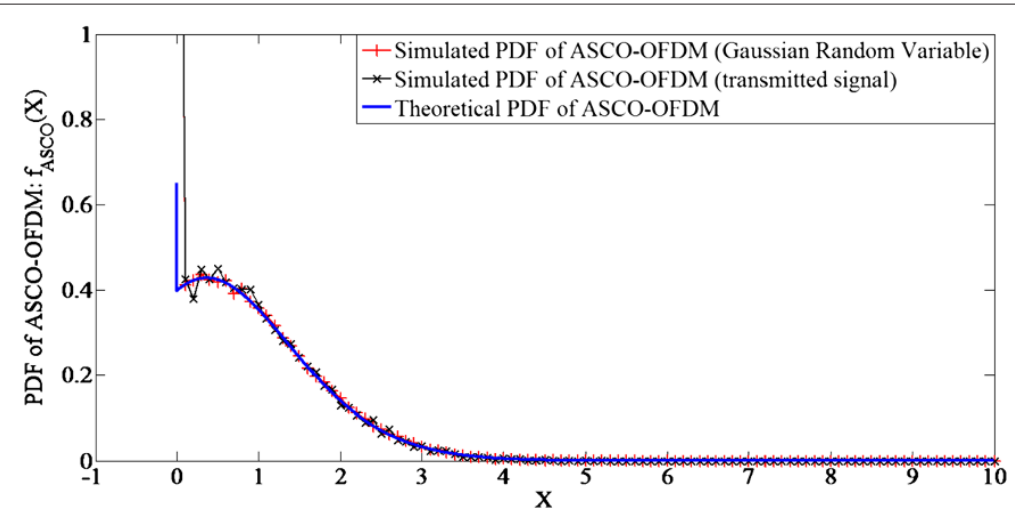

Figure 3 Theoretical and simulated PDF of ASCO-OFDM.

which is given by $\sigma_{D}=E\left\{x_{\text {even }}^{2}\right\}$. The PDF of ACO-OFDM signals is called the clipped Gaussian distribution, and it is only determined by $\sigma_{A}$. However, the PDF of DCO-OFDM signals is affected by $\sigma_{D}$ and $B_{\mathrm{DC}}$. Thus, the performance of ADO-OFDM partially depends on the DC bias level, $B_{\mathrm{DC}}$.

\subsection{The PDF of ASCO-OFDM}

In the ASCO-OFDM modulation scheme, $x_{\text {odd }}^{i, c}, x_{\text {odd }}^{j, c}$, $x_{\text {even }}^{c n}$, and $x_{\text {even }}^{c p}$ are all real clipped signals. $x_{\text {odd }}^{i, c}$ and $x_{\text {odd }}^{j, c}$ are asymmetrically clipped (ACO-OFDM signals) while $x_{\text {even }}^{c n}$ and $x_{\text {even }}^{c p}$ are symmetrically clipped (SCO-OFDM signals). We assume that they all follow a clipped Gaussian distribution, which is given by:

$$
f(x)=\frac{1}{\sqrt{2 \pi} \sigma} e^{-\frac{x^{2}}{2 \sigma^{2}}} u(x)+\frac{1}{2} \delta(x)
$$

where $\sigma$ is the standard deviation of the unclipped signal, such as $x_{\text {odd }}^{i}, x_{\text {odd }}^{j}$, or $x_{\text {even }}$. Here, we consider that $x_{\text {odd }}^{i}, x_{\text {odd }}^{j}$, and $x_{\text {even }}$ are modulated by the same constellation, so they have the same standard deviation $\sigma$. By using the clipped Gaussian distribution, we can obtain the PDF of ASCO-OFDM signals. Assuming $\alpha \in x_{\text {odd }}^{i, c}$ and $\beta \in x_{\text {even }}^{c n}(\alpha, \beta \geq 0), \chi=\alpha+\beta$ is the random variable of $x_{\mathrm{ASCO}}^{i}$. The PDF of $x_{\mathrm{ASCO}}^{i}$ is the convolution of $f_{x_{\text {odd }}^{i, c}}(\alpha)$ and $f_{x_{\text {even }}^{c n}}(\beta)$, which is given by:

$$
f(\chi)=f_{x_{\text {odd }}^{i, c}}(\alpha) \otimes f_{x_{\text {even }}^{c n}}(\beta)
$$

where:

$$
f_{x_{\text {odd }}^{i, c}}(\alpha)=\frac{1}{\sqrt{2 \pi} \sigma} e^{-\frac{\alpha^{2}}{2 \sigma^{2}}} u(\alpha)+\frac{1}{2} \delta(\alpha)
$$

and:

$$
f_{x_{\text {even }}^{c n}}(\beta)=\frac{1}{\sqrt{2 \pi} \sigma} e^{-\frac{\beta^{2}}{2 \sigma^{2}}} u(\beta)+\frac{1}{2} \delta(\beta)
$$

Thus, the PDF of $x_{\mathrm{ASCO}}^{i}$ is given by:

$$
f(\chi)=\left[\frac{1}{2 \sqrt{\pi} \sigma} e^{-\frac{\chi^{2}}{4 \sigma^{2}}} \operatorname{erf}\left(\frac{\chi}{2 \sigma}\right)+\frac{1}{\sqrt{2 \pi} \sigma} e^{-\frac{\chi^{2}}{2 \sigma^{2}}}\right] u(\chi)+\frac{1}{4} \delta(\chi)
$$

with mean $2 \sigma / \sqrt{2 \pi}$ and variance $(\pi-1) \sigma^{2} / \pi \cdot \operatorname{erf}(x)$ is the error function of $x$ and the PDF curve is shown in Figure 3. The calculation steps of Equation 27 are shown in Appendix 3.

The theoretical and simulated PDFs of ASCO-OFDM signals have been shown and compared in Figure 3. The theoretical result is given by Equation 27, which is the solid curve in the figure. Since the real bipolar signals at the output of an IFFT has a Gaussian distribution, we model $x_{\text {odd }}^{i}, x_{\text {odd }}^{j}$, and $x_{\text {even }}$ as Gaussian signals to generate the transmitted ASCO-OFDM signals. This simulated result is given by the plus solid curve. In order to obtain the PDF of signals generated by the ASCO-OFDM modulation scheme, we use a Monte Carlo experiment to get the statistical ASCO-OFDM signals. This simulated PDF is shown as a cross solid curve. Comparing these three curves, they closely match each other.

\subsection{Average bit rate/normalized bandwidth}

For both ADO-OFDM and ASCO-OFDM, $2 N-2$ complex symbols are modulated onto the spectrum with length $2 N$; hence, the normalized bandwidths for both are given by $N /(N-1)$. In ADO-OFDM, symbols carried on the odd and even subcarriers are drawn from different constellations because odd subcarriers are modulated by ACO-OFDM while even subcarriers are modulated by DCO-OFDM. The average bit rate of ADO-OFDM can be calculated by:

$$
R_{b, \mathrm{ADO}-\mathrm{OFDM}}=\left(\log _{2} C_{\mathrm{ACO}}+\log _{2} C_{\mathrm{DCO}}\right) / 2
$$

where $C_{\mathrm{ACO}}$ and $C_{\mathrm{DCO}}$ are the constellation sizes of ACO-OFDM symbols and DCO-OFDM symbols, respectively. Therefore, the average bit rate/normalized 
bandwidth of ADO-OFDM is given by $\left(\left(\log _{2} C_{\mathrm{ACO}}+\right.\right.$ $\left.\left.\log _{2} C_{\mathrm{DCO}}\right) / 2\right) /(N /(N-1))$. In order to improve the power efficiency of ADO-OFDM, DCO-OFDM symbols usually are drawn from small constellations to reduce the DC bias level. For ASCO-OFDM, all information carried in the even subcarriers is separated into two consecutive sub-blocks, $x_{\mathrm{ASCO}}^{i}$ and $x_{\mathrm{ASCO}}^{j}$. Thus, half of the information in $x_{\text {even }}$ is transmitted by the even components of one sub-block, $x_{\mathrm{ASCO}}^{i}$ or $x_{\mathrm{ASCO}}^{j}$. Therefore, the average bit rate of ASCO-OFDM for one sub-block can be obtained by:

$$
R_{b, \mathrm{ASCO}-\mathrm{OFDM}}=\left(\log _{2} C_{\mathrm{ACO}}+0.5 \log _{2} C_{\mathrm{SCO}}\right) / 2
$$

and the average bit rate/normalized bandwidth is given by $\left(\left(\log _{2} C_{\mathrm{ACO}}+0.5 \log _{2} C_{\mathrm{SCO}}\right) / 2\right) /(N /(N-1))$, where $C_{\mathrm{ACO}}$ and $C_{\mathrm{SCO}}$ are similarly defined. By applying different constellations to the odd and even subcarriers of ADO-OFDM and ASCO-OFDM, the average bit rate/normalized bandwidths for both are compared in Table 1.

\subsection{Optical power/bit}

In order to find out the accurate optical power per bit, $P_{\text {opt }} /$ bit, for both ADO-OFDM and ASCO-OFDM, we sum each value in the transmitted optical signal without adding the cyclic prefix. The total optical power of the transmitted ADO-OFDM signal is computed by:

$$
P_{\mathrm{opt}, \mathrm{ADO}}=\sum_{n=0}^{2 N-1} x_{\mathrm{ADO}}(n)
$$

The DC bias added in DCO-OFDM comes from Table 2. For ASCO-OFDM, the transmitted optical signal has two

\begin{tabular}{|c|c|c|c|}
\hline & ADO-OFDM & & ASCO-OFDM \\
\hline ACO 4-QAM & 2 & ACO 4-QAM & 1.5 \\
\hline DCO 4-QAM & & SCO 4-QAM & \\
\hline ACO 16-QAM & 3 & ACO 16-QAM & 2.5 \\
\hline DCO 4-QAM & & SCO 4-QAM & \\
\hline ACO 64-QAM & 4 & ACO 64-QAM & 3.5 \\
\hline DCO 4-QAM & & SCO 4-QAM & \\
\hline ACO 16-QAM & 4 & ACO 16-QAM & 3 \\
\hline DCO 16-QAM & & SCO 16-QAM & \\
\hline ACO 64-QAM & 5 & ACO 64-QAM & 4 \\
\hline \multirow[t]{3}{*}{ DCO 16-QAM } & & SCO 16-QAM & \\
\hline & & ACO 64-QAM & 4.5 \\
\hline & & SCO 64-QAM & \\
\hline
\end{tabular}
consecutive sub-blocks, $x_{\mathrm{ASCO}}^{i}$ and $x_{\mathrm{ASCO}}^{j}$. Therefore, the
Table 2 The list of ADO-OFDM constellation modified from reference [9] is used in Figures 4 and 5

\begin{tabular}{ccc}
\hline Symbol & ADO-OFDM constellation size & DC bias level \\
\hline A & ACO-OFDM 4-QAM, DCO-OFDM 4-QAM & $5.5 \mathrm{~dB}$ \\
\hline B & ACO-OFDM 16-QAM, DCO-OFDM 4-QAM & $5.1 \mathrm{~dB}$ \\
\hline C & ACO-OFDM 64-QAM, DCO-OFDM 4-QAM & $4.3 \mathrm{~dB}$ \\
\hline D & ACO-OFDM 16-QAM, DCO-OFDM 16-QAM & $7.3 \mathrm{~dB}$ \\
\hline E & ACO-OFDM 64-QAM, DCO-OFDM 16-QAM & $6.6 \mathrm{~dB}$ \\
\hline
\end{tabular}

total optical power of the transmitted ASCO-OFDM signal is given by:

$$
P_{\mathrm{opt}, \mathrm{ASCO}}=\sum_{n=0}^{2 N-1} x_{\mathrm{ASCO}}^{i}(n)+x_{\mathrm{ASCO}}^{j}(n)
$$

Considering the input of ADO-OFDM, a block of ( $N$ 1) $\times 1$ complex symbols is divided into two parts for ACO-OFDM and DCO-OFDM modulation. The $N / 2$ complex symbols are modulated onto the odd subcarriers by ACO-OFDM while the $N / 2-1$ complex symbols are applied into the even subcarriers for generating DCOOFDM signals. Therefore, the total transmitted bits for ADO-OFDM are given by:

$$
T_{b, \mathrm{ADO}-\mathrm{OFDM}}=(N / 2) \log _{2} C_{\mathrm{ACO}}+(N / 2-1) \log _{2} C_{\mathrm{DCO}}
$$

For ASCO-OFDM, the input block consisting of $3 N / 2-1$ complex symbols is separated into three parts. Two blocks of $N / 2$ complex symbols are modulated by ACO-OFDM to generate $x_{\text {odd }}^{i, c}$ and $x_{\text {odd }}^{j, c}$. One block of $N / 2-1$ complex symbols is modulated by SCO-OFDM to generate $x_{\text {even }}^{c n}$ and $x_{\text {even }}^{c p}$. Therefore, the total transmitted bits for ASCOOFDM are given by:

$$
T_{b, \mathrm{ASCO}-\mathrm{OFDM}}=2(N / 2) \log _{2} C_{\mathrm{ACO}}+(N / 2-1) \log _{2} C_{\mathrm{SCO}}
$$

\section{Simulation results}

The results of optical power per bit for ADO-OFDM and ASCO-OFDM are compared in Figure 4. Five constellation combinations denoted by $\mathrm{A}$ to $\mathrm{E}$, which are listed in Table 2, are applied to odd subcarriers (ACOOFDM) and even subcarriers (DCO- or SCO-OFDM) of two schemes. ADO-OFDM is represented by squares, and ASCO-OFDM is represented by stars. Star F represents ASCO-OFDM with 64-QAM symbols on all subcarriers. If two systems are modulated by the same constellation combination, the bit rate/normalized bandwidth of ASCO-OFDM is less than that of ADO-OFDM. However, ASCO-OFDM requires much less optical power for each bit than ADO-OFDM does due to no DC bias added in the SCO-OFDM modulation. Although 


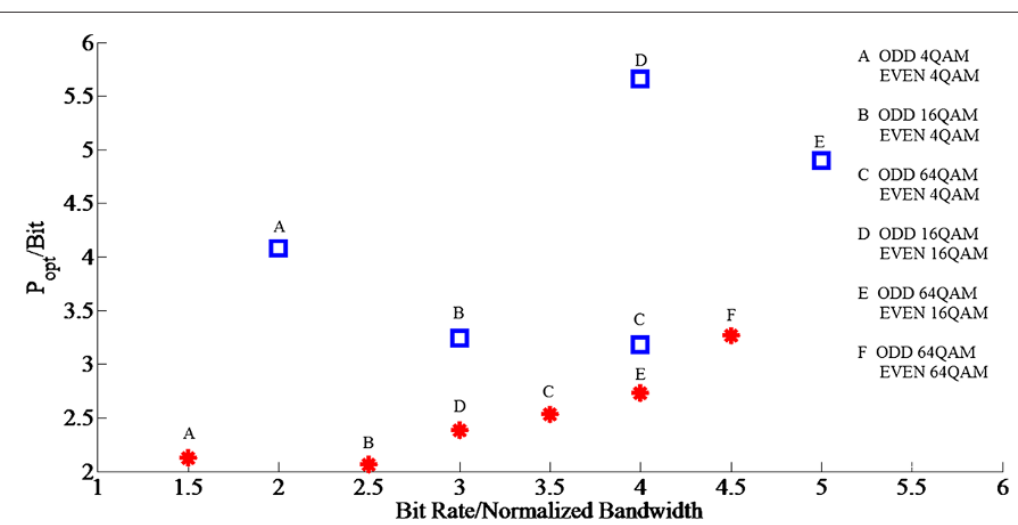

Figure 4 Optical power/bit versus bit rate/normalized bandwidth is shown for ADO-OFDM and ASCO-OFDM with different constellation combinations.

the bit rate/normalized bandwidth of ASCO-OFDM is lower, we can use larger constellation combinations to achieve the same bit rate with that of ADO-OFDM. When the same bit rate/normalized bandwidth is achieved, for example square $B$ vs. star $D$ and squares $C$ and $D$ vs. star E, the ASCO-OFDM exhibits more optical power efficiency than ADO-OFDM does. Therefore, ASCOOFDM is more optical power efficient compared to ADOOFDM. Moreover, if the required optical power per bit of ASCO-OFDM is close to that of ADO-OFDM, the bit rate/normalized bandwidth of ASCO-OFDM improves, which is shown as squares $B$ and $C$ vs. star $F$.

Figure 5 shows the SER of ADO-OFDM and ASCOOFDM applied with different constellation combinations. We use the same marker to represent the same constellation combination. A solid curve represents ASCO-OFDM while a dashed curve represents ADO-OFDM. In the figure, it is obvious that ASCO-OFDM has better SER performance than ADO-OFDM does in the same constellation combination case. Also, some specific comparison pairs should be clarified. Comparing the solid circle curve to the dashed plus curve, ASCO-OFDM with 164QAM has almost the same SER performance as 4-4QAM
ADO-OFDM, but ASCO-OFDM requires much less optical power and has a higher bit rate as shown in Figure 4. When achieving the same bit rate/normalized bandwidth, ASCO-OFDM outperforms ADO-OFDM in terms of the SER performance as well as optical power efficiency, which is shown as a solid square curve (ASCO-OFDM with 16-16) and a dashed circle curve (ADO-OFDM with 16-4). The solid cross curve (ASCO-OFDM with 64-16) and the dashed down-triangle curve (ADO-OFDM with 64-4) have the same performance in terms of SER and bit rate/normalized bandwidth, but the optical power per bit required by ASCO-OFDM is much less than that of ADOOFDM. Finally, we point out that ASCO-OFDM (solid hexagon) is better than ADO-OFDM (dashed square) in all aspects because it has lower optical power per bit, higher bit rate/normalized bandwidth, and better SER performance.

\section{Conclusions}

In this paper, we propose a novel OFDM scheme called ASCO-OFDM for IM/DD optical wireless systems. A conventional ACO-OFDM is applied to modulate odd subcarriers, and the novel modulation scheme,

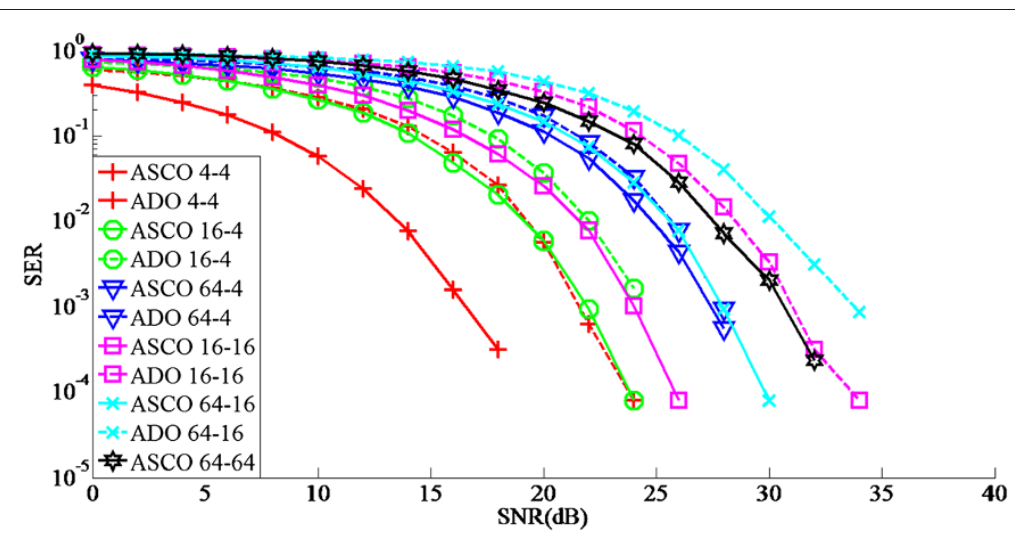

Figure 5 Comparison of SER (Symbol error rate) versus SNR for ADO-OFDM and ASCO-OFDM with different constellation combinations. 
SCO-OFDM, is used to modulate even subcarriers. For SCO-OFDM, we successfully adopt two consecutive subblocks to transmit the symbols on the even subcarriers without adding DC bias. Since the odd components and the even components of two systems can be separately detected, different constellation combinations are taken into account for modulation. Compared to ADO-OFDM, our novel optical system exhibits better SER performance and requires lower optical power per bit. For these reasons, we believe that ASCO-OFDM is the most attractive choice for IM/DD optical wireless systems.

\section{Appendix 1}

The IDFT of a length $2 N$ frequency domain sequence $X(k)$ is defined by:

$$
x(n)=\frac{1}{2 N} \sum_{k=0}^{2 N-1} X(k) \exp \left(\frac{j 2 \pi n k}{2 N}\right)
$$

In order to present Equation 34 by the sum of the odd subcarriers and the even subcarriers, we use a new index $l$ instead of $k$,

$$
\begin{aligned}
x(n)= & \frac{1}{2 N} \sum_{l=0}^{N-1} X(2 l) \exp \left(\frac{j 2 \pi n 2 l}{2 N}\right) \\
& +\frac{1}{2 N} \sum_{l=0}^{N-1} X(2 l+1) \exp \left(\frac{j 2 \pi n(2 l+1)}{2 N}\right)
\end{aligned}
$$

If the odd subcarriers of $X(k)$ are set to zero, then the second term of Equation 35 is zero. Thus, $x(n)$ is given by:

$$
x(n)=\frac{1}{2 N} \sum_{l=0}^{N-1} X(2 l) \exp \left(\frac{j 2 \pi n 2 l}{2 N}\right)
$$

Then, we calculate $x(n+N)$. Substituting index $n+N$ into Equation 36, we have:

$$
\begin{aligned}
x(n+N) & =\frac{1}{2 N} \sum_{l=0}^{N-1} X(2 l) \exp \left(\frac{j 2 \pi(n+N) 2 l}{2 N}\right) \\
& =\frac{1}{2 N} \sum_{l=0}^{N-1} X(2 l) \exp \left(\frac{j 2 \pi n 2 l}{2 N}\right) \exp \left(\frac{j 2 \pi N 2 l}{2 N}\right)
\end{aligned}
$$

Note that the second exponential term can be simplified to $\exp (j 2 \pi l)$, which is equal to one. Therefore, we prove that an OFDM symbol converted from only even subcarriers satisfies the property that $x(n)=x(n+N)$.

\section{Appendix 2}

Supposing $x(n)$ is an SCO-OFDM signal of length $2 N$, then the DFT of $x(n)$ is given by:

$$
\begin{aligned}
X(k)= & \sum_{n=0}^{2 N-1} x(n) \exp \left(\frac{-j 2 \pi n k}{2 N}\right) \\
= & \sum_{\substack{n=0 \\
x(n)>0}}^{N-1}\left[x(n) \exp \left(\frac{-j 2 \pi n k}{2 N}\right)\right. \\
& \left.+x(n+N) \exp \left(\frac{-j 2 \pi(n+N) k}{2 N}\right)\right] \\
& +\sum_{\substack{n=0 \\
x(n)<0}}^{N-1}\left[x(n) \exp \left(\frac{-j 2 \pi n k}{2 N}\right)\right. \\
& \left.+x(n+N) \exp \left(\frac{-j 2 \pi(n+N) k}{2 N}\right)\right]
\end{aligned}
$$

The exponential term $\exp \left(\frac{-j 2 \pi(n+N) k}{2 N}\right)$ is equal to $\exp \left(\frac{-j 2 \pi n k}{2 N}\right) \exp (-j \pi k)$, and the value of $\exp (-j \pi k)$ is given by:

$$
\exp (-j \pi k)= \begin{cases}1, & k \text { is even } \\ -1, & k \text { is odd }\end{cases}
$$

By using the property of $x(n)=x(n+N)$ and substituting Equation 39 into Equation 38, we obtain that:

$$
X(k)= \begin{cases}2 \sum_{\substack{n=0 \\ x(n)>0}}^{N-1} x(n) \exp \left(\frac{-j 2 \pi n k}{2 N}\right) & \\ +2 \sum_{\substack{n=0 \\ x(n)<0}}^{N-1} x(n) \exp \left(\frac{-j 2 \pi n k}{2 N}\right) & k \text { is even } \\ 0 & k \text { is odd }\end{cases}
$$

After clipping, the SCO-OFDM symbols on the odd and even subcarriers are, respectively, shown as:

$$
X^{C}(k)= \begin{cases}2 \sum_{\substack{n=0 \\ x(n)>0}}^{N-1} x(n) \exp \left(\frac{-j 2 \pi n k}{2 N}\right) & k \text { is even } \\ 0 & k \text { is odd }\end{cases}
$$

We notice that the symbols on the odd subcarriers are always equal to zero, and the symbols on the even subcarriers lose the second summation in Equation 40. Thus, we prove that the SCO-OFDM clipping noise only affects even subcarriers without distorting the data on the odd subcarriers. 


\section{Appendix 3}

The derivation of the PDF of ASCO-OFDM signals is shown as:

$$
\begin{aligned}
f(\chi)= & \int_{-\infty}^{\infty} f_{x_{\text {odd }}, c}(\alpha) f_{x_{\text {even }}^{c \text { c }}}(\chi-\alpha) d \alpha \\
= & \int_{-\infty}^{\infty}\left[\frac{1}{\sqrt{2 \pi} \sigma} e^{-\frac{\alpha^{2}}{2 \sigma^{2}}} u(\alpha)+\frac{1}{2} \delta(\alpha)\right] \\
& \cdot\left[\frac{1}{\sqrt{2 \pi} \sigma} e^{-\frac{(\chi-\alpha)^{2}}{2 \sigma^{2}}} u(\chi-\alpha)+\frac{1}{2} \delta(\chi-\alpha)\right] d \alpha \\
= & \int_{-\infty}^{\infty} \frac{1}{2 \pi \sigma^{2}} e^{-\frac{\alpha^{2}}{2 \sigma^{2}}} e^{-\frac{(\chi-\alpha)^{2}}{2 \sigma^{2}}} u(\alpha) u(\chi-\alpha) d \alpha \\
& +\int_{-\infty}^{\infty} \frac{1}{2 \sqrt{2 \pi} \sigma} e^{-\frac{\alpha^{2}}{2 \sigma^{2}} u(\alpha) \delta(\chi-\alpha) d \alpha} \\
& +\int_{-\infty}^{\infty} \frac{1}{2 \sqrt{2 \pi} \sigma} e^{-\frac{(\chi-\alpha)^{2}}{2 \sigma^{2}}} \delta(\alpha) u(\chi-\alpha) d \alpha \\
& +\int_{-\infty}^{\infty} \frac{1}{4} \delta(\alpha) \delta(\chi-\alpha) d \alpha \\
= & {\left[\frac{1}{2 \sqrt{\pi} \sigma} e^{-\frac{\chi^{2}}{4 \sigma^{2}}} \operatorname{erf}\left(\frac{\chi}{2 \sigma}\right)+\frac{1}{\sqrt{2 \pi} \sigma} e^{-\frac{\chi^{2}}{2 \sigma^{2}}}\right] u(\chi)+\frac{1}{4} \delta(\chi) }
\end{aligned}
$$

\section{Competing interests}

The authors declare that they have no competing interests.

Received: 5 September 2014 Accepted: 15 December 2014

Published: 15 January 2015

\section{References}

1. J Armstrong, OFDM for optical communications. IEEE J. Lightwave Technol. 27(3), 189-204 (2009)

2. K Acolatse, Y Bar-ness, SK Wilson, Novel techniques of single-carrier frequency-domain equalization for optical wireless communications. EURASIP J. Adv. Signal Process. 2011, 4 (2011)

3. K Acolatse, Y Bar-ness, SK Wilson, SCFDE with space-time coding for IM/DD optical wireless communication. IEEE Wireless Commun. Netw. Conf. (WCNC), 1694-1699 (2011)

4. Z Ghassemlooy, S Rajbhandari, W Popoola, Optical Wireless Communications: System and Channel Modeling with MATLAB, 1st ed. (CRC Press, New York, 2012)

5. Y Sun, Bandwidth-efficient wireless OFDM. IEEE J. Sel. Areas Commun. 19(11), 2267-2278 (2001)

6. O Gonzalez, R Perez-Jimenez, S Rodriguez, J Rabadan, A Ayala, Adaptive OFDM system for communications over the indoor wireless optical channel. IEEE Proc.-Optoelectronics. 153, 139-144 (2006)

7. J Armstrong, BJC Schmidt, D Kalra, HA Suraweera, AJ Lowery, in Proc. IEEE Global Telecommunications Conference. Performance of asymmetrically clipped optical OFDM in AWGN for an intensity modulated direct detection system (San Francisco, CA, USA, Nov. 27-Dec. 1 2006), pp. 1-5

8. SK Wilson, J Armstrong, Digital modulation techniques for optical asymmetrically-clipped OFDM. IEEE Wireless Commun. Netw. Conf, 538-542 (2008)

9. SD Dissanayake, J Armstrong, Comparison of ACO-OFDM, DCO-OFDM and ADO-OFDM in IM/DD systems. J. Lightwave Technol. 31(7), 1063-1072 (2013)

10. B Ranjha, M Kavehrad, Hybrid asymmetrically clipped OFDM-based IM/DD optical wireless system. IEEE/OSA J. Opt. Commun. Netw. 6(4), 387-396. 014

11. H Elgala, TDC Little, P-OFDM: Spectrally efficient unipolar OFDM. Opt. Fiber Commun Conf. Exhibition (OFC), 1-3 (2014)

12. J Armstrong, B Schmidt, Comparison of asymmetrically clipped optical OFDM and DC-biased optical OFDM in AWGN. IEEE Commun. Lett. 12(5), 343-345 (2008)
13. J Armstrong, AJ Lowery, Power efficient optical OFDM. Electron Lett. 42(6), 370-371 (2006)

14. H Elgala, R Mesleh, H Haas, Indoor broadcasting via white LEDs and OFDM. IEEE Trans. Consumer Electron. 55(3), 1127-1134 (2009)

15. H Elgala, R Mesleh, H Haas, in Proceedings of the 6th international conference on Wireless and Optical Communications Networks (WOCN '09). A study of LED nonlinearity effects on optical wireless transmission using OFDM (Piscataway, NJ, USA, 2009), pp. 388-392

16. SK Wilson, J Armstrong, Transmitter and receiver methods for improving asymmetrically-clipped optical OFDM. IEEE Trans. Wireless Commun. 8(9), 4561-4567 (2009)

17. E Katz, A Laufer, Y Bar-ness, in 46th Annual Conference on Information Sciences and Systems. A new improved-performance decoding technique for Asymmetrically-Clipped Optical-OFDM (Princeton, NJ, USA, 2012), pp. 1-6

18. N Wu, Y Bar-ness, An improved performance decoding technique for asymmetrically and symmetrically clipped optical (ASCO)-OFDM. Appear in Asilomar Conference on Signals, Systems, and Computers (2014)

19. DJF Barros, SK Wilson, JM Kahn, Comparison of orthogonal frequency-division multiplexing and pulse-amplitude modulation in indoor optical wireless links. IEEE Trans. Commun. 60(1), 153-163 (2012)

20. C Liang, B Krongold, J Evans, Performance analysis for optical OFDM transmission in short-range IM/DD systems. J. Lightwave Technol. 30, 974-983 (2012)

21. X Li, R Mardling, J Armstrong, in Proceedings of IEEE International Conference Communications. Channel capacity of IM/DD optical communication systems and of ACO-OFDM (Glasgow, UK, 2007), pp. 2128-2133

doi:10.1186/1687-6180-2015-3

Cite this article as: Wu and Bar-Ness: A novel power-efficient scheme asymmetrically and symmetrically clipping optical (ASCO)-OFDM for IM/DD optical systems. EURASIP Journal on Advances in Signal Processing 2015 2015:3.

\section{Submit your manuscript to a SpringerOpen ${ }^{\circ}$ journal and benefit from:}

- Convenient online submission

- Rigorous peer review

- Immediate publication on acceptance

- Open access: articles freely available online

- High visibility within the field

- Retaining the copyright to your article

Submit your next manuscript at $\gg$ springeropen.com 